\title{
The identification of challenges in tissue collection for biomarker studies: the Q-CROC-03 neoadjuvant breast cancer translational trial experience
}

Adriana Aguilar-Mahecha ${ }^{1}$, Josiane Lafleur ${ }^{1}$, Manuela Pelmus ${ }^{2}$, Carole Seguin ${ }^{3}$, Cathy Lan ${ }^{1}$, Federico Discepola ${ }^{3}$, Bojan Kovacina ${ }^{3}$, Rosa Christodoulopoulos ${ }^{4}$, Ombretta Salvucci ${ }^{5}$, Catalin Mihalcioiu ${ }^{6}$, Josée- Anne Roy ${ }^{7}$, André Robidoux ${ }^{8}$, Elizabeth A Marcus ${ }^{9}$, Gerald Batist ${ }^{1,10}$ and Mark Basik ${ }^{1}$

${ }^{1}$ Department of Oncology, Lady Davis Institute, McGill University, Montreal, QC, Canada; ${ }^{2}$ Department of Pathology, Jewish General Hospital, Montreal, QC, Canada; ${ }^{3}$ Department of Radiology, Jewish General Hospital, Montreal, McGill University, Montreal, QC, Canada; ${ }^{4}$ Clinical Research Unit, Jewish General Hospital, Montreal, McGill University, Montreal, QC, Canada; ${ }^{5}$ Laboratory of Cellular Oncology, Center for Cancer Research, National Cancer Institute, National Institutes of Health, Bethesda, MD, USA; ${ }^{6}$ McGill University Hospital Center, Montreal, QC, Canada; ${ }^{7}$ Hôpital du Sacre Coeur, Montreal, QC, Canada; ${ }^{8}$ Centre Hospitalier de l'Université de Montreal, Montreal, QC, Canada; ${ }^{9} J o h n$ H. Stroger Jr. Hospital, Chicago, IL, USA and ${ }^{10}$ Quebec Clinical Research Organization in Cancer, Montreal, QC, Canada

One of the major challenges in biomarker development is the collection of tumor tissue of adequate quality for analysis. A prospective clinical trial was initiated to collect tissues from triple negative breast cancers prior to and after neoadjuvant chemotherapy in order to study the mechanisms of chemoresistance. Sixty patients had pre-chemotherapy biopsies performed by either a surgeon or a radiologist, while those with residual tumor after chemotherapy had research-only biopsies and/or surgical samples collected in liquid nitrogen, RNA-later and formalin. We examined each core for tumor cellularity, stromal content, and necrosis after which, RNA and DNA extraction was performed. We found that biopsies collected with ultrasound guidance were more likely to contain tumor than those collected by the surgeon. Patient reluctance to undergo research-only biopsies after chemotherapy was not a problem. Pre-chemotherapy tumor biopsies frequently did not contain any tumor cells $(15 \%)$ or did not have $\geq 50 \%$ tumor content $(63 \%)$. Indeed, $50 \%$ of patients had at least 2 pre-chemotherapy core biopsies with $<50 \%$ tumor content. After chemotherapy, $30 \%$ of biopsy or surgical samples in patients with incomplete response did not contain any tumor. Finally, RNA-later not only made histopathological assessment of tumor content difficult, but yielded less DNA than fresh snap frozen samples. We recommend that high-quality tissue procurement can be best accomplished if at least three image-guided core biopsies be obtained per sample, each of these cores be examined for tumor cellularity and that at least some of them be freshly snap frozen in liquid nitrogen.

Modern Pathology (2017) 30, 1567-1576; doi:10.1038/modpathol.2017.82; published online 28 July 2017

With the advent of personalized treatments and the incorporation of biomarkers in clinical oncological practice, tumor tissue sampling is now increasingly

Correspondence: Dr M Basik, MDCM, Department of Oncology McGill University, Jewish General Hospital, 3755 Côte SteCatherine Lady Davis Institute, Montreal H371E2, QC, Canada.

E-mail: mark.basik@mcgill.ca

Received 28 March 2017; revised 23 May 2017; accepted 25 May 2017; published online 28 July 2017 requested as a mandatory component of modern clinical trials. Tissues are collected in order to perform correlative science research and/or to assign patients to treatment groups depending on the expression of specific biomarkers. ${ }^{1}$ In fact, the collection of serial tissue specimens before, during and after treatment provides an excellent opportunity to study the mechanisms of response or resistance to drugs and to identify predictive biomarkers for response to these drugs, which have 
the potential to reduce cancer care costs and thus improve accessibility to expensive treatments. ${ }^{2,3}$

Although establishing an infrastructure for tissue collection in the context of clinical trials is challenging to implement, it is required in order to ensure the collection of sufficient high-quality material to guarantee the success of molecular analysis. ${ }^{4,5}$ The most critical factors that can impact on the results of molecular analyses include tissue quality, the amount of tumor contained within the tissue collected as well as the yield and integrity of the genomic material extracted. ${ }^{6-8}$ To minimize preanalytical variability, the appropriate method of sample preservation and processing needs to be defined and standardized up-front according to the type of downstream molecular profiling to be performed. Although the quality of tissues may best be preserved if they are collected fresh and frozen, many clinical centers do not have the requisite capacity for such a collection, thus making the use of tissue preservatives necessary. This is especially the case if tissue must be shipped to a central laboratory. However, there still is a paucity of data dealing with the impact of the method of preservation on the histological and molecular quality of the biospecimen, especially within a clinical trial.

In the present study, we describe our experience with the collection and processing of research biopsies and surgical specimens in the context of a clinical trial designed to identify biomarkers of resistance to standard chemotherapy in triple negative breast cancer patients (NCT01276899). We herein discuss the limitations encountered during sample collection and we present the results of our qualitative analysis on histology, nucleic acids and tissue heterogeneity.

\section{Materials and methods}

\section{Patient Population}

The Quebec Clinical Research Organization in Cancer-03 trial (Q-CROC-03 trial, NCT01276899) enrolled patients from August 2010 to December 2013 in five centers in Canada and one site in the United States. Women with triple negative breast cancer were eligible to participate in the neoadjuvant arm of the trial if the primary tumor was T2 or greater, had no evidence of systemic metastatic disease and were to receive neoadjuvant chemotherapy as per local standard of care. Triple negative receptor status was defined as per the American Association of Clinical Oncology guidelines at first (ER/PR expression $<1 \%$ and HER2 negative), although upon review of original pathology, 10 patients were found to have ER/PR expression $<10 \%$ but $>1 \%$ and four patients had ER/PR $>10 \%$. All patients are included in the present analysis. The study was approved by each institution's Research Ethics Board and complied with local ethics guidelines; every patient provided written informed consent.

\section{Sample Collection}

For each patient, 3-4 needle core biopsies (14 or 16 gauge) were collected for research purposes prior to chemotherapy and again prior to surgery. Surgery was performed 3-4 weeks after completion of neoadjuvant treatment ( 4-6 months duration). 3-4 fragments of residual tumor from the surgical specimen were collected in the department of pathology when available, according to the pathologist on service (Supplementary Figure S1). Patients had an ultrasound performed prior to treatment and following the completion of the chemotherapeutic regimen and clinical evaluations performed before, mid-way and after chemotherapy. The residual cancer burden index was determined as previously reported by Symmans et al. ${ }^{9}$

\section{Sample Processing}

Needle core biopsies or pieces from residual tumors were preserved in the following manner. At each time point, two samples were freshly snap frozen in liquid nitrogen, and one sample was placed in $1 \mathrm{ml}$ of RNA-later (Qiagen, Toronto, ON, Canada) and immediately frozen. Frozen samples were stored at each center in $-80^{\circ} \mathrm{C}$ freezers and shipped on dry ice in batches to a central laboratory (Jewish General Hospital, Montreal, QC, Canada). The fourth sample was placed in $10 \%$ formalin and immediately shipped at $4^{\circ} \mathrm{C}$ to the central lab for paraffin embedding (Supplementary figure S2). If the sample was collected on Friday, the formalin specimen was kept at $4^{\circ} \mathrm{C}$ and shipped the following Monday. Biospecimens were processed at the central laboratory following standard operating procedures for histopathological analysis and nucleic acid extraction. ${ }^{10}$ All frozen samples were embedded in optimal cutting temperature medium (Surgipath; Leica biosystems, Concord, ON, Canada) as previously reported. ${ }^{10}$ Samples that were collected in RNA-later underwent two consecutive washes in ice-cold 1x RNAse-free phosphate-buffered saline (Fisher Scientific, Mississauga, ON, Canada) prior to embedding in optimal cutting temperature medium following standard operating procedures previously published by our group. ${ }^{10}$ Cryosections ( $5 \mu \mathrm{m}$ thick) were cut with the cryostat temperature set to $-25^{\circ}$ or $-30^{\circ}$ depending on the size of the breast cancer tissue, cut sections were stained with hematoxylin and eosin. A breast pathologist reviewed the stained slides and provided the percentage of tumor content, stroma and necrosis for each sample. The tumorcontaining area was circled by the pathologist so that samples with low tumor cellularity could be enriched by macrodissection for nucleic acid extraction. ${ }^{10}$ For each sample for which nucleic acid extraction was performed, pictures were taken before 
and after macrodissection to document the tumor area used, stained slides of matching blocks were scanned using the Aperio ImageScope system (Leica Biosystems, ON, Canada).

Simultaneous extraction of DNA and RNA was performed using the AllPrep DNA/RNA/miRNA Universal Kit (Qiagen). The ratio of the absorbance at 260 and $280 \mathrm{~nm}$ as well as DNA and RNA concentrations were measured with the NanoDrop spectrophotometer (Thermo Fisher, Waltham, MA, USA). RNA integrity was measured with the Bioanalyzer 2100 (Agilent Technologies, Santa Clara, CA, USA). Double stranded DNA concentration was measured with PicoGreen ds DNA Assay kit (Thermo Fisher).

The Advanced Tissue Management Application kindly provided by the Canadian Tissue Repository Network was used as the biorepository database.

\section{Statistical Analysis}

We performed Pearson correlations, $\chi^{2}$-test for Count data in $\mathrm{R}$ to identify any significant difference $(P<0.05)$ in nuclear morphological preservation and biospecimen heterogeneity.

Two-tailed unpaired student t-tests were used to compare DNA and RNA yields between type of samples (biopsy vs surgery) and processing methods (RNA-later vs liquid nitrogen). Statistical significance is defined as $P<0.05$.

\section{Results}

\section{Biospecimen Collection}

The Q-CROC-3 clinical trial was designed to study mechanisms of drug resistance and to identify candidate biomarkers of resistance to chemotherapy in primary and metastatic triple negative breast cancer. The approach was to compare prechemotherapy and post-chemotherapy tumor samples using genomic techniques. The neoadjuvant study recruited 60 patients with primary triple negative breast cancer, although four patients were taken off study due to ER/PR levels $>10 \%$ observed after slide re-evaluation. Since the present study focuses on the evaluation of the quality of samples collected, all 60 patients were included in our analysis. Two patients did not have surgery due to the detection of distant metastasis during treatment and one patient was lost to follow up. For three patients, pre-chemotherapy biopsies and surgical samples were retrieved retrospectively from the breast biobank at the Jewish General Hospital. In these cases, no post-chemo biopsies were obtained. Biopsies were performed either under ultrasound guidance or by the treating breast surgeon. Of the 60 patients, 28 participated in an ongoing breast biopsy biobank program at the Jewish General Hospital in which additional research biopsies are obtained at the time of diagnostic breast mass biopsy. In these cases, since the same standard operating procedures were used as for the Q-CROC-3 study, these research biopsies served as the pre-chemotherapy biopsy samples and no additional biopsy procedure was necessary. It is noteworthy that Q-CROC-03 patients participating in the breast biopsy biobank program provided an average of seven cores (four cores for diagnosis and three cores for research) at the time of diagnostic breast mass biopsy. One patient refused to have the additional three research biopsies performed. The remaining 31 patients had an additional pre-chemotherapy biopsy performed only for research and 3-4 cores were collected following breast cancer diagnosis. One of these patients had additional biopsies both in the breast and lymph node. Independent analysis of our patient cohort demonstrated that patients were highly cooperative and willing to undergo repetitive procedures to provide tissue for research purposes. ${ }^{11}$ Twenty $(65 \%)$ of these additional biopsies were performed under ultrasound guidance and the rest (11 or $35 \%$ ) by the breast surgeon on palpable masses. There were no serious adverse events related to the biopsy procedures.

Samples were collected in liquid nitrogen, RNAlater and formalin following established standard operating procedures and all clinical centers complied with instructions provided and respected the times suggested for preservation. The average time from sample collection to preservation was 1-2 min for all snap frozen biopsies and 56 min for surgical specimens (including sample transit time to their pathology department). Samples in formalin were received at the central laboratory within 24hrs of shipping and processed within $24 \mathrm{~h}$ of reception. All samples shipped on dry ice were received frozen at the central lab.

The rate of pathological complete response, defined as the absence of invasive disease in the breast and axillary lymph nodes after neoadjuvant treatment, was $43 \%$ in our cohort. This is similar to pathological complete response rates previously reported for triple negative breast cancer patients. ${ }^{12}$ In order to preserve uniform conditions of sample collection, we decided to collect research-only biopsies prior to surgery, depending on the clinical and/or radiological presence of tumor upon completion of chemotherapy. The goal was to minimize the impact of cold ischemia on the quality of the genomic data and to be able to compare a core needle biopsy pre-chemo with a core needle biopsy post-chemo. Recognizing the extra burden placed on the research centers and the patients in performing these biopsies, we did not make them mandatory. Of the total cohort, 54 patients were considered for postchemotherapy biopsy. Eleven of these 54 patients $(20 \%)$ did not have radiological or clinical evidence of residual tumors, and were not scheduled for biopsy. Of the remaining 43 who had evidence of residual tumors by imaging, 21 (39\% of the total) did not undergo biopsy for the following reasons: patient 

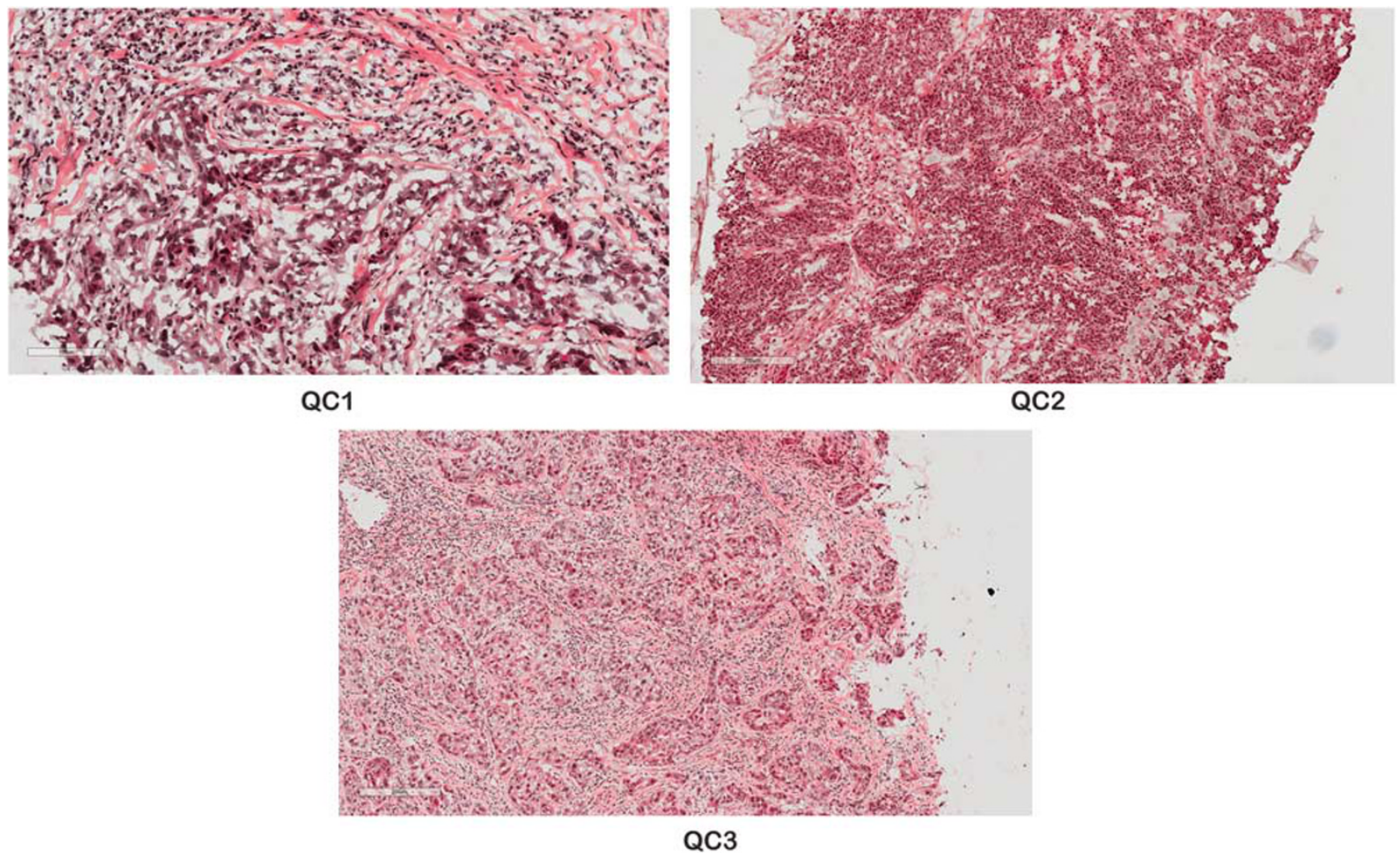

Figure 1 Hematoxylin and eosin staining of breast cancer tissues with QC1, QC2, and QC3 nuclear morphology preservation. QC1, poor preservation of chromatin, nuclear shape and distinction of mitotic figures such that accurate grading is not possible; QC2, moderate preservation such that some nuclear detail and mitotic figures can only be distinguished with difficulty; QC3, good to excellent preservation of nuclear features. Slides were scanned with Aperio ImageScope system at $20 \times$ magnification.

refusal $(n=4)$, lack of local capacity to perform the second biopsy $(n=8)$, inability to schedule the biopsy in time prior to surgery $(n=6)$, development of metastases prior to surgery $(n=2)$ and persistent post-chemotherapy toxicity $(n=1)$. Post-chemo biopsies were performed on average 20 days after the end of treatment (range 3-41 days) and 22 days prior to surgery (range 5-58 days). Of the 22 patients undergoing post-chemotherapy biopsies who had residual tumor masses on post-treatment ultrasound, 7 (32\%) had no residual tumor both at the post-chemotherapy biopsy and at the time of surgery, suggesting that the presence of a residual mass at post-chemotherapy ultrasound is not a very sensitive predictor of residual tumor at surgery. In one patient with pathological complete response, the ultrasoundguided biopsy revealed tumor cells, implying that the post-chemotherapy biopsy had removed all tumor cells from the residual tumor bed.

Fragments of residual tumors were collected at the time of surgery and residual cancer burden scores were calculated for surgical specimens. ${ }^{9}$ In two centers, pathologists on service at the time of surgery refused to provide tissues. In total, we obtained surgical tissue samples from 27 patients; remarkably, 26 of these patients were recruited at the main clinical site (Jewish General Hospital). Nine patients were eventually determined to have pathological complete response or residual cancer burden score I, as expected, none of those surgical samples contained tumor cells after pathological evaluation. In total, we collected 207 pre-chemo and 91 postchemo core breast biopsies, 12 post-chemo lymph node biopsies and 105 residual tumor specimens throughout the whole study. A subset of these samples was processed for molecular profiling.

\section{Tissue Histological Quality}

A pathologist (MP) reviewed stained slides for 296 of the samples collected. The quality of tissue specimens was assessed by looking at nuclear morphological preservation, which was graded according to a pre-determined scale (Figure 1) and used as a measure of histological quality of tissues collected under different conditions. ${ }^{13}$ We found that despite washing out the RNA-later prior to tissue embedding, cryosection was more difficult to perform on samples placed in RNA-later and frozen. These tissue sections often contained areas with missing tissue and the tissue remaining on the slide was often of poor histological quality. In fact, $45 \%$ of RNA-later samples had poor nuclear morphology preservation (QC1), rendering pathological evaluation challenging. Fresh snap frozen samples had better 
histological quality with $75 \%$ of samples with moderate (QC2) or good nuclear morphology (QC3). As expected, formalin fixation and paraffin embedding resulted in the best preservation of nuclear morphology (Figure 2).

\section{Tissue Heterogeneity}

We evaluated 2-4 cores per patient time point for the proportion of the core area that consisted of tumor, and the proportion of tumor cells, stromal cells and necrosis within the tumor areas. As shown in Figure 3, the content of each core biopsy was quite heterogeneous in serial samples collected from the same patient. Similar heterogeneity was present in surgical specimens (data not shown). For prechemotherapy biopsies, $15 \%$ of biopsy cores had no detectable tumor cells within them and 17 of 60 patients $(28 \%)$ had at least one pre-chemotherapy core without any tumor in it. Only two patients (Neo01 and Neo-18) had no tumor on all pre-chemo biopsy cores. For the post-chemo biopsies in patients with residual tumor on final pathology (i.e. not with pathological complete response), $30 \%$ of biopsy cores had no tumor cells within them, and 7 of 15 patients $(47 \%)$ had at least one core without tumor cells. For the surgical samples, $19(30 \%)$ of surgical samples with residual tumor had no detectable tumor cells within them (Table 1). The above findings strongly suggest that a minimum of $2-3$ cores is required to get enough tissue to perform any meaningful molecular study in this clinical context. In order to qualify for molecular analyses, we set the threshold for cellularity within the tumor area to $\geq 50 \%$, which was adequate to enable high-quality downstream molecular analyses including next generation sequencing without the need for macrodissection. ${ }^{14}$ Overall, $42 \%$ of evaluated individual tumor samples met the minimum threshold of at least $50 \%$ tumor cellularity $37 \%$ pre-chemo core biopsy samples, $33 \%$ post-chemo core biopsy samples and $54.7 \%$ of surgical specimen-derived fragments) (Table 1). For the pre-chemotherapy biopsies, 50\% (30 of 60) of patients had biopsies in which at least 2 cores had either no tumor or $<50 \%$ tumor cells. Again, these results support the need for the collection of multiple cores to ensure that at least one core will contain enough tumor cellularity for molecular profiling. In fact, since we collected at least 3 biopsy cores per time point for each patient, we managed to obtain at least one core with $\geq 50 \%$ tumor cellularity for 43 out of 60 patients.

Whenever tumor cellularity was less than $50 \%$ and when the pathologist could clearly delineate the section containing tumor cells on the slide, macrodissection was performed to enrich for tumor cellularity (Supplementary Figure S3). With macrodissection it was possible to salvage 21 of 104 samples that had not initially passed the $50 \%$

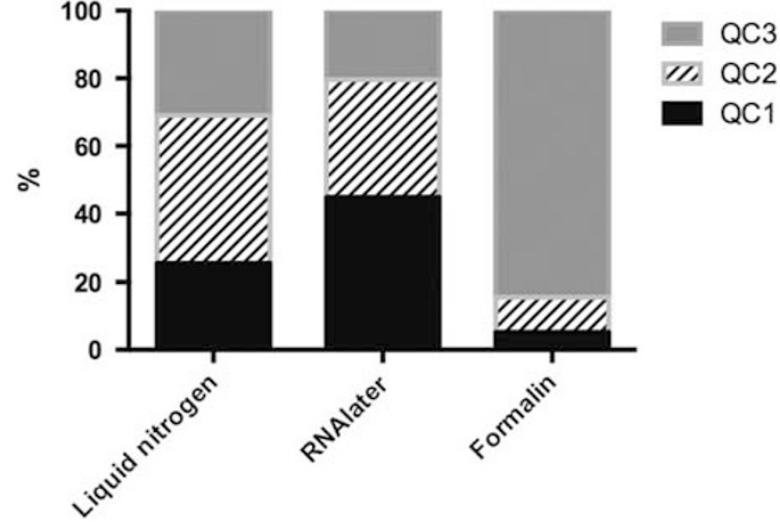

Figure 2 Quality of nuclear morphological preservation (QC1, QC2, and QC3) as evaluated by a breast pathologist in samples collected under different conditions (RNA-later, fresh snap frozen (liquid nitrogen), and formalin fixed paraffin embedded). ${ }^{*} P<0.05$ (Pearson's $\chi^{2}$-test $=65.573, \mathrm{df}=4, P$-value $=1.949 \mathrm{e}-13$ ).

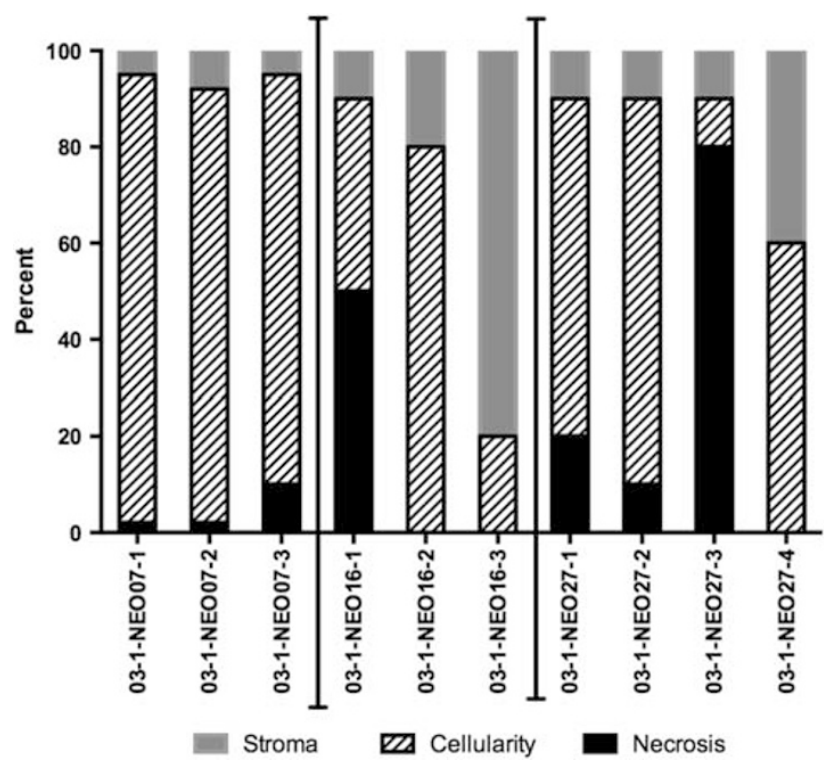

Figure 3 Heterogeneity of 3-4 pre-chemotherapy core biopsies collected from the same tumors in three different patients (Neo07, Neo16, and Neo27). The percentage of stroma, tumor cellularity and necrosis were evaluated by a pathologist.

cellularity threshold. We frequently observed that the amount and shape of the tissue section on the stained slide was not representative of the amount and shape of tissue present in the matching block. This recurrent problem was often due to the presence of normal breast tissue composed of fat that does not adhere to the slide and also to the asymmetric position of the sample during the embedding procedure. When the tissue section showed a low proportion of tumor area on the slide, we used the pathologist's evaluation as a guide to perform macrodissection of the tissue in the block to match the stained slide (Figure 4). This procedure was performed on $40 \%$ of the samples that were processed for nucleic acid extraction. 
Table 1 Tumor cellularity in biopsies and surgical specimens

\begin{tabular}{lcrrrr}
\hline Collection & Not evaluable & no tumor cells & $<50 \%$ tumor & $\geq 50 \%$ tumor & Total evaluated \\
\hline Pre-chemo therapy biopsies & $4.6 \%(n=8)$ & $14.5 \%(n=25)$ & $43.7 \%(n=75)$ & $37.2 \%(n=64)$ \\
Post-chemo therapy biopsies & $5 \%(n=3)$ & $30 \%(n=18)$ & $31.7 \%(n=19)$ & $33.3 \%(n=20)$ \\
Surgical specimens & 0 & $29.6 \%(n=19)$ & $15.6 \%(n=10)$ & $54.7 \%(n=35)$ & 172 \\
& & & & 60 \\
\hline
\end{tabular}

In those cases when the surgeon performed the prechemotherapy biopsy without ultrasound guidance (33 core biopsies evaluated from 11 patients), 18\% of cores were not evaluable by the pathologist because of insufficient tissue, $24 \%$ had no tumor cellularity and only $27 \%$ met the $\geq 50 \%$ threshold. Four post-chemo therapy biopsies were collected from two patients without ultrasound guidance, and each of these biopsy cores contained $>50 \%$ tumor cellularity. It is of note that these two patients presented tumors that grew during neoadjuvant treatment and were highly accessible for a non-ultrasound-guided biopsy procedure. For ultrasound-guided biopsies, far fewer were not evaluable ( $1 \%$ of pre-chemotherapy cores and $5 \%$ of post-chemotherapy cores). In these biopsies, tumor cells were absent in 12 and $32 \%$ of pre-chemo and post-chemo biopsies respectively, while only $39 \%$ of pre-chemotherapy biopsies and $29 \%$ of post-chemotherapy samples met the $\geq 50 \%$ threshold (Figure 5 and Supplementary Table S1). Post-chemotherapy biopsies and surgical fragments collected from patients who had pathological complete response were not included in this analysis.

We then assessed the effect of tissue preservation method on the pathologist's ability to assess cellularity. Although not statistically significant, we observed that the proportion of samples that was not evaluable was twice as high in RNA-later samples compared to snap frozen samples and four times higher when compared to parafin embedded samples. However, the percentage of samples meeting the $\geq 50 \%$ cellularity threshold was very similar across processing methods $\left(\chi^{2}\right.$-test $P=0.64$; Table 2 ).

\section{Nucleic Acid Yields and Quality}

Simultaneous purification of DNA and total RNA was performed on 93 samples (71 liquid nitrogen and 21 RNA-later) that met the cellularity threshold. We chose dual extraction to maximize the yield from each biospecimen and to minimize the bias that may be introduced by intra-tumor heterogeneity when analyzing genomic and transcriptomic data. The relationship between DNA and RNA yield per biospecimen with type of collection (biopsy vs surgery) and sample processing (RNA-later vs fresh snap frozen (liquid nitrogen)) is depicted in Figure 6. DNA and RNA yields tended to be lower in biopsies compared to surgical specimens regardless of collection method. RNA-later frozen samples yielded significantly less DNA from surgical samples when compared to liquid nitrogen samples $(P<0.001)$
(Figure 6a). Although not statistically significant $(P=0.22$ for biopsies and $P=0.09$ for surgical specimens), the average yield of RNA was lower in RNAlater samples $(4.92 \mu \mathrm{g}$ in biopsies and $17.2 \mu \mathrm{g}$ in surgical tissues) compared to liquid nitrogen samples $(17.5 \mu \mathrm{g}$ in biopsies and $56.52 \mu \mathrm{g}$ in surgical tissues; Figure 6b). The quality of RNA as assessed by the RNA Integrity Number was on average 7.6 (range 3.4-9.1) and 6.2 (range 1.8-8.7) for liquid nitrogen and RNA-later samples, respectively. It is of note that quality of RNA was not affected in samples that were macrodissected (data not shown).

\section{Discussion}

The genomics revolution has enabled the development and use of targeted therapies as well as brought about an understanding of mechanisms of drug resistance resulting in novel predictive biomarkers. ${ }^{15-17}$ Modern clinical trials frequently mandate biopsies and stratify and/or enroll patients depending on tissue biomarker measurements. ${ }^{1,18,19}$ Although analysis and interpretation of whole genome data remains challenging, the weakest and frequently forgotten link in the process of genomic biomarker development and correlative science analysis is the organization required to ensure the collection of tissue biopsies able to provide highquality nucleic acids in sufficient amounts. ${ }^{4,5,20}$ For this reason a considerable amount of energy and time must be consecrated to the development and application of standard operating procedures for the collection and processing of precious tumor material prior to initiating patient accrual. Cognizant of these issues, in preparation for the Q-CROC-3 clinical trial, we performed extensive analyses evaluating multiple facets of tissue collection and processing, and developed standard operating procedures, some of which have been made publicly available in the National Cancer Institute-Standard Operating Procedure library (https://brd.nci.nih.gov/ $\mathrm{brd} /$ ). In addition, we also configured a data collection system composed of case report forms for each procedure (biospecimen collection and storage, tissue embedding, histopathological assessment and nucleic acid extraction) where pre-analytical variables such as time of collection, time of preservation, time of storage, temperature of shipping and arrival could be recorded. The Canadian Tumor Repository Network's Advanced Tissue Management application allowed us to keep track of the hundreds of 


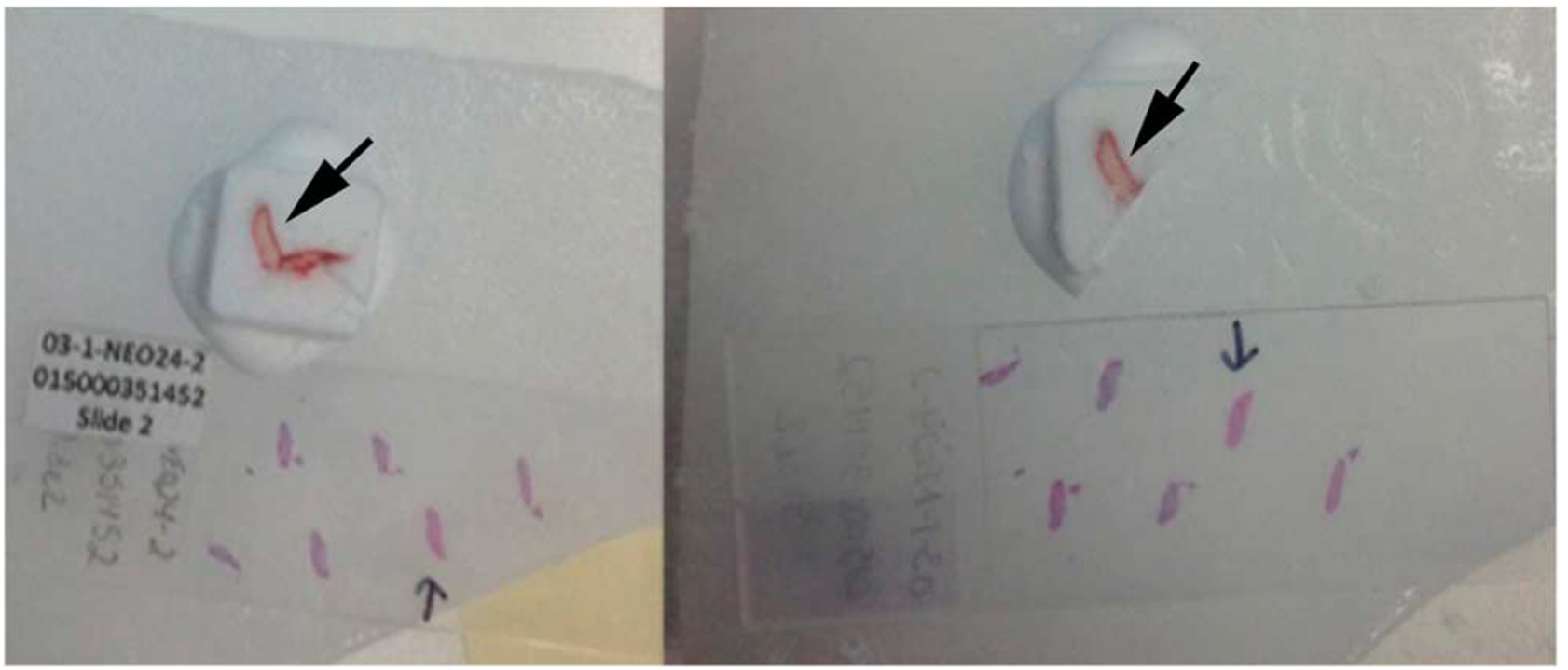

Figure 4 (a) Picture of block of breast biopsy embedded in optimal cutting temperature medium, only one portion of the biopsy is represented on the slide stained with hematoxyllin and eosin (arrow). (b) Macrodissection was performed to isolate the tissue that was represented on the stained slide.

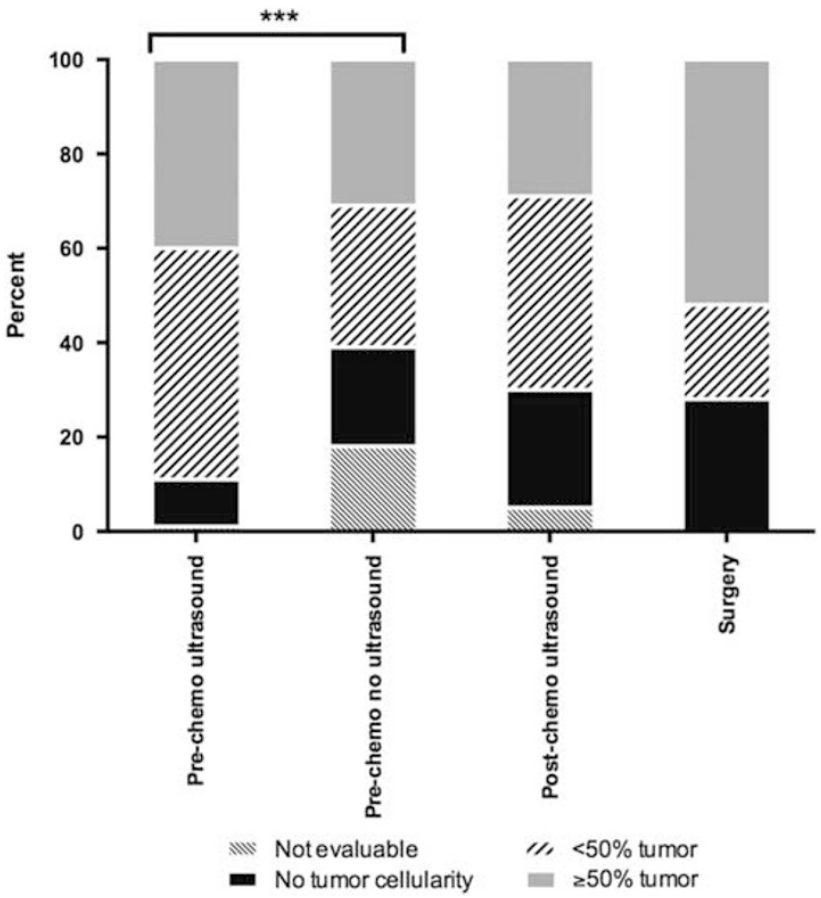

Figure 5 Tissue composition of pre-chemo biopsies collected with $(n=139)$ and without $(n=33)$ ultrasound, post-chemo biopsies collected with ultrasound $(n=56)$ and of tissue fragments collected at the time of surgery $(n=64)$. Pre-chemotherapy biopsies collected with ultrasound are statistically different from those collected by the surgeon (with no ultrasound). Pearson's $\chi^{2}$-test $P$-value $=7.492 \mathrm{e}-06$.

biospecimens collected and data associated with each biospecimen including location, derivatives (tissue blocks, slides, DNA, RNA, and so on) and usage enabling fast data retrieval for the present study. We now present the results of our efforts at
Table 2 Tumor content evaluation in samples collected in RNA-later, liquid nitrogen or formalin

\begin{tabular}{lccc}
\hline & RNA-later & Liquid nitrogen & Formalin \\
\hline $\begin{array}{l}\text { Not evaluable } \\
\text { Only normal }\end{array}$ & $6.9 \%(n=4)$ & $3.4 \%(n=6)$ & $1.5 \%(n=1)$ \\
tissue & $20.7 \%(n=12)$ & $20.1 \%(n=35)$ & $23.4 \%(n=15)$ \\
$\quad<50 \%$ tumor & $36.2 \%(n=21)$ & $36.2 \%(n=63)$ & $31.2 \%(n=20)$ \\
$\begin{array}{l}\text { content } \\
\geq 50 \% \text { tumor }\end{array}$ & $36.2 \%(n=21)$ & $40.2 \%(n=70)$ & $43.8 \%(n=28)$ \\
content & & & \\
\hline
\end{tabular}

standardizing tissue collection and processing within a multicenter biomarker-driven clinical trial.

The context in which we operated is that of the neoadjuvant clinical trial in operable breast cancer, which has been proposed as a context of choice for the discovery and validation of biomarkers in breast cancer. Indeed, the accessibility of larger breast tumors to both image-guided and surgical biopsy both pre- and post-treatment provides an ideal situation to enable highly efficient tissue biopsy accrual and quality. However, analyzing our results proved to be an eye opening experience.

First, we found that obtaining informed consents from patients for research-only biopsies, even after chemotherapy prior to surgery was rarely an issue. At the Jewish General Hospital we were able to benefit from a mechanism whereby any patient with a suspicious breast mass over $2 \mathrm{~cm}$ is automatically consented for extra research biopsies at the time of diagnostic breast biopsy. This involves a 15-30 min discussion with a clinical nurse who meets with the patient prior to the biopsy at the Breast Imaging Center. In this way, we avoid second research-only 
a

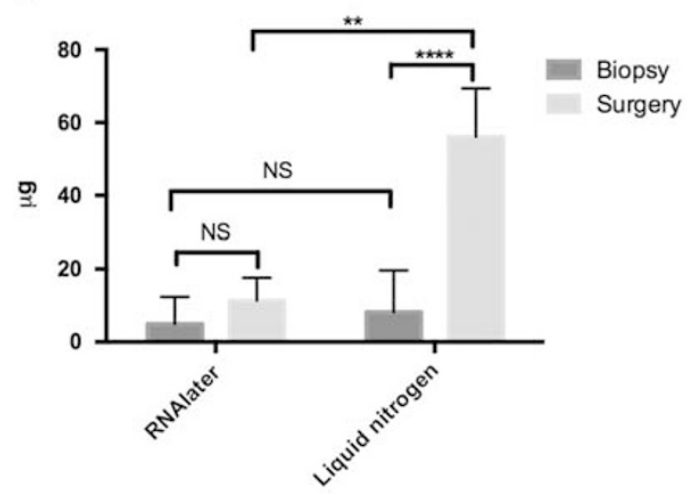

b

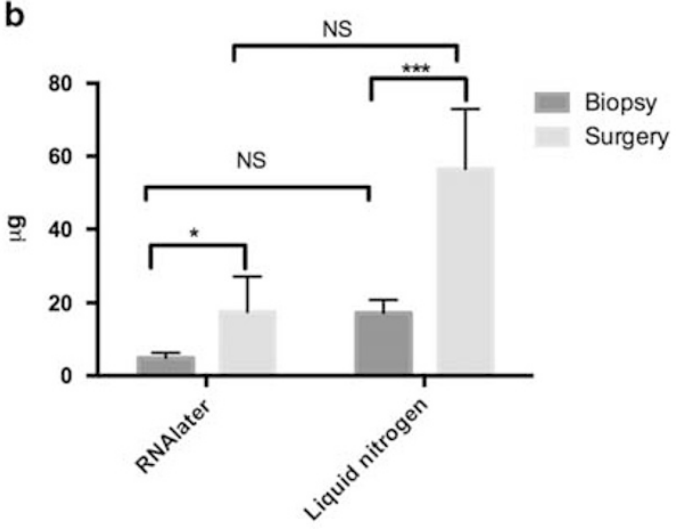

Figure 6 DNA (a) and RNA (b) yields from biopsies $(n=80)$ and surgical specimens $(n=16)$ freshly frozen (liquid nitrogen) or in RNA-later. DNA and RNA levels were measured by NanoDrop spectrophotometer. Groups were analyzed for statistical significance $(P<0.05)$ with two-tailed unpaired Student's $t$-tests.

biopsies in a large proportion of breast cancer patients eligible for neoadjuvant clinical trials.

In order to standardize the pre/post treatment biopsies as much as possible and to avoid the issues related to warm and cold ischemia of surgical samples, ${ }^{21,22}$ centers were encouraged to obtain the post-chemotherapy tissue sample in the same way as the pre-chemotherapy biopsy, i.e. as a biopsy and not as surgery-derived tissue fragments. Interestingly, the collection of post-chemotherapy biopsies proved to be difficult in a substantial number of cases, but not because of patient reticence. In cases where the ultrasound did detect a residual mass after chemotherapy $(n=34)$, the lack of the capacity to repeat a biopsy or the inability to time the biopsy prior to surgery resulted in not performing a second biopsy in 14 cases (33\%), notably greater than the rate of patient refusal (4 cases, 9\%). The lack of a postchemo biopsy was often compensated by the collection of a post-chemo specimen at the time of surgery for many of our patients.

Based on our previous experience, ${ }^{10}$ we decided to assess every biopsy and surgical sample for tumor cellularity, stromal content and necrosis. This process required the cooperation and availability of a dedicated breast pathologist and, although timeconsuming, it ensured that the samples collected for molecular analysis were of optimal tumor cellularity. We found tissue histological quality to be best preserved in formalin fixed paraffin embedded samples and least in samples frozen in RNA-later. The assessment of tumor cellularity was more challenging in the RNA-later specimens and close to $7 \%$ of biospecimens in RNA-later were not evaluable. We and others had shown that washing of RNA-later samples prior to tissue embedding and cryosectioning improves the histological quality of breast and liver tissues. ${ }^{10,23}$ However, snap frozen samples retain better histology and are easier to evaluate by the pathologist in our experience.

We found that less than half of all specimens contained the required $50 \%$ tumor cellularity and that there was considerable heterogeneity amongst the samples from each patient's tumor. Since 50\% (30 of 60) of patients had pre-chemotherapy biopsies in which at least 2 cores had either no tumor or $<50 \%$ tumor cells, we recommend that a minimum of 3 cores be obtained for research purposes even when biopsying easily accessible primary breast tumors. Surprisingly, only 55\% of surgically derived fragments provided by the pathology department after visual selection of a tumor region met this not overly strict criterion. This result reinforces our concerns about examining each and every specimen for tumor cellularity and quality and also the need for collection of multiple cores at each given time point. Due to the fact that more than half of the samples collected were below the 50\% threshold cellularity for molecular profiling, molecular profiling platforms had to be prioritized. In fact, samples originally collected for proteomic analysis (one of freshly snap frozen samples) were instead used for nucleic acid extraction for next generation sequencing studies to compensate for the low tumor cellularity. We frequently resorted to macrodissection in order to obtain sufficient concentrations of tumor cells for genomic analysis and this allowed us to save a significant number of samples without compromising RNA quality.

We also found that $39 \%$ of pre-chemotherapy samples obtained without ultrasound guidance by a breast surgeon did not contain any tumor or were inadequate, compared to only $11 \%$ when the radiologist performed the biopsy. Although we were not comparing the 2 methods on the same tumorswhich would have been ethically impossible-these results strongly suggest to us that the performance of image-guided biopsies to obtain adequate tumor tissue for correlative science studies is preferable to non-image-guided biopsies even in palpable breast tumors.

Finally we compared the DNA and RNA yield between flash frozen samples placed in RNA-later or not, as well as in biopsy vs surgical samples, and 
found that DNA yield was significantly better in freshly frozen samples than in RNA-later samples and that RNA yield was also somewhat higher although not significantly so in the freshly frozen samples compared to RNA-later samples. Very few studies have compared DNA yields in RNA-later and snap frozen samples. For instance, a recent study reported lower DNA yields in chorionic villi collected in RNA-later compared to snap frozen samples. ${ }^{24}$ During sample processing we did notice a harder consistency of RNA-later tissues which rendered manual homogenization more difficult, with this difference being more noticeable in larger surgical tumor fragments (compared to biopsy specimens), which may explain their lower DNA and RNA yields. Lower RNA yields observed in RNAlater compared to freshly snap frozen samples are consistent with our previous observations in liver specimens. ${ }^{10}$

In conclusion, we present the real-time application and performance of standard operating procedures for the collection and processing of fresh tissue samples within the context of a multicenter clinical trial in early stage breast cancer. Although several difficulties were revealed throughout the study, the adoption of quality control measures allowed us to ensure that only samples of optimal quality were selected for molecular analyses. Therefore, for all clinical trials for which high-quality samples are being collected we recommend to: (i) verify each sample (meaning every core biopsy or surgically derived fragment) for the presence of tumor; (ii) encourage fresh snap frozen sample collection if possible; (iii) develop infrastructures capable of collecting and processing serial biopsies in clinical trial settings and storing its associated data; (iv) collect at least three core biopsies for research purposes; and (v) encourage image-guided biopsies of palpable masses. Such principles may help avoid the collection and use of poor quality tissue samples, which would endanger the interpretation of results from expensive multicenter trials in which biomarker research is funded. We believe that our experience may be of assistance to clinical trialists planning trials in which biomarkers are integrated or mandated as part of the protocol.

\section{Acknowledgments}

We are grateful to all the patients who participated in this study and to all the site coordinators and radiologists of the Q-CROC-03 study. We thank: Lilian Cannetti and Naciba Belimane for services provided by the Jewish General Hospital Pathology Core, Kathleen Klein for biostatistical services and Nicolas Luc for his constant support with the Advancer Tissue Management database. The breast biobank infrastructure was supported by the FRQS Réseau Recherche Cancer axe Cancer du Sein. This study was supported by Genome Quebec with cofunding from Quebec Breast Cancer Foundation, McGill University and Jewish General Hospital Foundation.

\section{Disclosure/conflict of interest}

The authors declare no conflict of interest.

\section{References}

1 Papadimitrakopoulou V, Lee JJ, Wistuba II, et al. The BATLE-2 study: a Biomarker-Integrated Targeted Therapy Study in previously treated patients with advanced non-small-cell lung cancer. J Clin Oncol 2016;34:3638-3647.

2 Daud AI, Wolchok JD, Robert C, et al. Programmed death-ligand 1 expression and response to the antiprogrammed death 1 antibody pembrolizumab in melanoma. J Clin Oncol 2016;34:4102-4109.

3 Assouline SE, Nielsen TH, Yu S, et al. Phase 2 study of panobinostat with or without rituximab in relapsed diffuse large B-cell lymphoma. Blood 2016;128: 185-194.

4 Aguilar-Mahecha A, Diaz Z, Buchanan M, et al. Making personalized medicine a reality: the challenges of a modern translational research biopsy-driven program in an academic setting: the Segal Cancer Center experience. J Med Pers 2011;9:104-111.

5 Basik M, Aguilar-Mahecha A, Rousseau C, et al. Biopsies: next-generation biospecimens for tailoring therapy. Nat Rev Clin Oncol 2013;10:437-450.

6 Bilge A, Hongxia S, Hui Y, et al. Global gene expression changes induced by prolonged cold ischemic stress and preservation method of breast cancer tissue. Mol Oncol 2014;8:717-727.

7 Dominic AP, Laura MA, Arran KT, et al. Tumour sampling method can significantly influence gene expression profiles derived from neoadjuvant window studies. Sci Rep 2016;6:29434.

8 Dolgin E, Shoddy biopsies deny cancer patients a shot at personalized treatment, available at: https:/www.stat news.com/2016/01/22/precision-medicine-cancer-biopsies 2016.

9 Symmans WF, Peintinger F, Hatzis C, et al. Measurement of residual breast cancer burden to predict survival after neoadjuvant chemotherapy. J Clin Oncol 2007;25:4414-4422.

10 Diaz Z, Aguilar-Mahecha A, Paquet ER, et al. Next-generation biobanking of metastases to enable multidimensional molecular profiling in personalized medicine. Mod Pathol 2013;26:1413-1424.

11 Abadie R, Kimmelman J, Lafleur J, et al. Consent for nondiagnostic research biopsies: a pilot study of participant recall and therapeutic orientation. IRB 2014;36:9-15.

12 Huober J, von Minckwitz G, Denkert C, et al. Effect of neoadjuvant anthracycline-taxane-based chemotherapy in different biological breast cancer phenotypes: overall results from the GeparTrio study. Breast Cancer Res Treat 2010;124:133-140.

13 Canadian Tissue Repository Network, Standard Operating Procedure 5.1001. Assessing Quality of Tissue Specimens, available at: http://www.rrcancer.ca/med 
ias/files/admin/sop/ctrnet-sop-5.1.001-e1.0-assessingquality-of-tissue-specimens.pdf.

14 Chen H, Luthra R, Goswami RS, et al. Analysis of Pre-Analytic Factors Affecting the Success of Clinical Next-Generation Sequencing of Solid Organ Malignancies. Cancers (Basel) 2015;7:1699-1715.

15 Bardelli A, Corso S, Bertotti A, et al. Amplification of the MET receptor drives resistance to anti-EGFR therapies in colorectal cancer. Cancer Discov 2013;3: 658-673.

16 Katayama R, Khan TM, Benes C, et al. Therapeutic strategies to overcome crizotinib resistance in nonsmall cell lung cancers harboring the fusion oncogene EML4-ALK. Proc Natl Acad Sci USA 2011;108: 7535-7540.

17 Shi H, Moriceau G, Kong X, et al. Melanoma wholeexome sequencing identifies (V600E)B-RAF amplification-mediated acquired B-RAF inhibitor resistance. Nat Commun 2012;3:724.

18 Park JW, Liu MC, Yee D, et al. Adaptive Randomization of Neratinib in Early Breast Cancer. N Engl J Med 2016;375:11-22.
19 Schwaederle M, Parker BA, Schwab RB, et al. Precision Oncology: The UC San Diego Moores Cancer Center PREDICT Experience. Mol Cancer Ther 2016;15:743-752.

20 Liu A. Developing an institutional cancer biorepository for personalized medicine. Clin Biochem 2014;47: 293-299.

21 Dash A, Maine IP, Varambally S, et al. Changes in differential gene expression because of warm ischemia time of radical prostatectomy specimens. Am J Pathol 2002;161:1743-1748.

22 Holzer TR, Fulford AD, Arkins AM, et al. Ischemic time impacts biological integrity of phospho-proteins in PI3K/Akt, Erk/MAPK, and p38 MAPK signaling networks. Anticancer Res 2011;31:2073-2081.

23 Ellis M, Davis N, Coop A, et al. Development and validation of a method for using breast core needle biopsies for gene expression microarray analyses. Clin Cancer Res 2002;8:1155-1166.

24 Pisarska MD, Akhlaghpour M, Lee B, et al. Optimization of techniques for multiple platform testing in small, precious samples such as human chorionic villus sampling. Prenat Diagn 2016;36:1061-1070.

Supplementary Information accompanies the paper on Modern Pathology website (http://www.nature.com/ modpathol) 